\title{
TI.129.1
}

Steward Program Proof of Concept Ends

- PDF: StewardProgramProofOfConceptEnds-2017-07-24.pdf

- Text: StewardProgramProofOfConceptEnds-2017-07-24.txt

\section{More Information}

\begin{tabular}{|l|l|}
\hline Repository ID & Tl.129.1 \\
\hline Persistent URL & http://doi.org/10.26869/TI.129.1 \\
\hline Title & Steward Program Proof of Concept Ends \\
\hline Authors & Ann West https://orcid.org/0000-0001-5484-6827 \\
\hline Sponsor & Internet2 Trust and Identity Executive Management \\
\hline Review & \\
\hline Status & Legacy \\
\hline Publish Date & July 24, 2017 \\
\hline DOI & $10.26869 /$ TI.129.1 \\
\hline Signature & \\
\hline Deprecated & No \\
\hline Future Review & \\
\hline Supersedes & \\
\hline Format & PDF, Text \\
\hline Related Docs & \\
\hline Development Location & \\
\hline IP Framework & \\
\hline Subject Tags & \\
\hline Notes & \\
\hline & \\
\hline
\end{tabular}

